\title{
Towards a democratic approach on public lighting: remote systems based on Metadesign
}

\section{SIGRADI2018 TECHNOPOLITICAS xxiicongresso da sociedadeiberoamericana de gráfica digital \\ 22th conference of the \\ iberoamerican society of digital graphics 07|08|09|novembro|2018 iau usp | são carlos | sp br}

\author{
Gabriela Correia Fernandes \\ Universidade Estadual de Londrina | Brazil | gabrielacorreia.fernandes@gmail.com
}

Prof. Dr. Rovenir Bertola Duarte

Universidade Estadual de Londrina | Brazil | rovenir@uel.br

Beatriz Ferreira de Oliveira

Universidade Estadual de Londrina | Brazil | oliveirafe.beatriz@gmail.com

Giovana Medri Striquer Souza

Universidade Estadual de Londrina | Brazil | giovanastriquer@gmail.com

\begin{abstract}
This research explores Metadesign(Giaccardi, 2003)as an innovative framework on the design of urban lighting systems. We hypothesize that a system based on this mode of design can bring breakthroughs that could cope with ill-defined problems in urban lighting design. Therefore, the aim is to propose alighting system in support of participation through interaction at use time. In this sense, by comprehending lighting infrastructures as sociotechnical environments, we believe Metadesign could cope with emergent needs arising in the context of personally meaningful activities andcould empower users to engage actively in their systems development and evolution.
\end{abstract}

Keywords: Metadesign; Participation at use time; Sociotechnical systems; Urban lighting design

\section{INTRODUCTION}

This paper describes part of an ongoing research that explores Metadesign(Giaccardi, 2003)as an innovative framework in the design of urban lighting systems. By putting the owners of problems in charge of the design while using the system, we hope to reach better solutions to problems even inside an ever-changing context of use. The design principles that address this approach will be outlined and followed by the description of the proposed system design. Our objective is to establish the scientific foundation for designing lighting systems open to participation at use time. We believe that comprehending lighting infrastructures as sociotechnical environments we could respond to emergent problems in the context of personally meaningful activities. With this purpose, users will be invited to collaborate actively in the process of continual development and evolution.

The Metadesign framework sees participation at use time as the source to achieve better solutions to problemsand to keep the system and the world in sync (Giaccardi \& Fischer, 2008). Through an intentional shift in the way systems are designed, we believe that is possible to support users as active contributors while they are using lighting infrastructures. The Information and Communication Technology (ICT) has been seen as the primary facilitator to the new emerging solutions to urban problems and also as the responsible for a new paradigm of interaction that opens the opportunity of building cities more democratically(Nabian \& Ratti, 2012)(Nabian \&
Ratti, 2013). Nevertheless, many experiences developed over the last few years that were based on ICT solutions, have encountered difficulties in responding to the problems proposed. In a broader perspective, the malfunctioning in these cases appears to be related to the lack of integration with the context and their dynamic, and not about technological inefficiency (Roche, Nabian, Kloeckl, \& Ratti, 2012).

We can consider a challenge both the operation of new technologies and the design of infrastructures in the current context. In the case of urban lighting infrastructures, for example, the influence of artificial light is so significant that it is capable of affecting our health and well-being (Gaston, Gaston, Bennie, \& Hopkins, 2014), and in a social context, is able to reflect on our behavior(Magielse, Hengeveld, \& Frens, 2013). Even though aware of its impact, local authorities have difficulties in designing such infrastructures. There is a gap in understanding how people engage with the infrastructure on a day-to-day basis, resulting in uncertainties that designers have a problem to overcome (Shaw, 2014).

All these issues create demands and raise debates about new patterns of interaction with urban lighting. It is undeniable that opportunities are rising from technology and that there is an urgency for designing systems more supportive to their users. Furthermore, to design lighting infrastructures that allow users to explore their personal 
preferences and desires, it is necessary to redefine the approach towards lighting systems (Offermans, 2016).

\section{PARTICIPATION AT USE TIME}

Within conventional design processes,two stagescanbe easily differentiated: design time and use time. The traditional design methodologies are primarily concerned with design time when a future is projected,and all design efforts move towards "the world as imagined". In current design practices this typically results in closed solutions based on decisions made at design time, and which users are incapable of modifying. At use time, users will use the designed object even if the system requires modification to fit their needs, because these needs were only partially foreseen at design time(Fischer \& Giaccardi, 2004;Fischer, 2010;Giaccardi \& Fischer, 2008). In traditional participatory approaches, users have an active role in contributing to design, but objects are still designed as complete artifacts at design time. The design is based on users' input, but it cannot be evolved to accommodate unforeseen needs(Gerhard Fischer, 2010).

Therefore, to vanish with this division, a new conceptual and methodological design framework is required. This framework is called Metadesign, a mode of design that is open to embrace problems that emerge at use time by engaging users to act as designers within in the process of co-creation and supported co-evolution of humans and technology (Giaccardi, 2003). This non-division between design time and use time in the design process configures a paradigm shift. To put users as owners of problems at use time means to engage them in problem-framing and problem-solving processes while using the system. It requires a design that is not only concerned about fitting better to users' needs, but that is also open to cope with unexpected problems focused on the use and also on participation(Fischer, 2010; Giaccardi \& Fischer, 2008).

To better understand why this approach can be seen as promising in responding to problems arising from the current context, it is necessary to outline the main design issues related to participation at use time inside Metadesign(Giaccardi, 2003)framework:

Anticipation issues: from Metadesign perspective, solving problems in design is a continuous process. Therefore it cannot be exclusively done at design time. The issue of anticipation is related to the limited ability of designers to anticipate users' needs, and it questions whether emerging problems at use time should be excluded from the design process.

- Participation issues: reflect on users' role within the design process, and how this affects the role of designers themselves. When compared to traditional participatory design approaches, the difference is that they are not concerned with designs that support structural changes, and processes of mutual evolution at use time. Though it is focused on users, traditional participation is configured as a way to increase the chances of responding better to their needs, but it ignores emerging situations.

- Sociotechnical issues: it looks at design from a sociotechnical perspective, and at its meaning in today's reality. It reflects on the relationship between humans and technologies and encloses the previous issues, questioning means and motivations for co-design practices (Giaccardi, 2003).

Therefore, based on Metadesign, the ongoing research will verify if this new mode of participatory design could answer to urban lighting problems raised in the current technological context. By proposing a system open to participation through interaction at use time, we will analyze the potential that Metadesign has to respond to the following issues: 1. anticipation - the need for infrastructures design to incorporate issues closer to their context and use; 2. participation - the construction of cities through a more democratic perspective; 3. socio-technical - the insertion of a user perspective into these systems.

\section{METADESIGN FRAMEWORK}

As a conceptual framework, Metadesign(Giaccardi, 2003)arises in the context of computational systems design supported by theoretical discussions in the field of interactive art, in which collaboration is seen as an opportunity for creativity and intelligence to emerge(Gerhard Fischer, Giaccardi, Eden, Sugimoto, \& $\mathrm{Ye}, 2005)$. Developed from a linguistic inheritance of the prefix meta, it refers to the idea of changes related to place, order or nature (Giaccardi, 2003; Giaccardi, 2005). Metadesign seeks the reflection of how to build sociotechnical systems that allow users to be creative and act as designers when desired, by incorporating emerging aspects of reality into the design process (Giaccardi \& Fischer, 2008). Already applied in the fields of Net Art and open source platforms, here we will examine if this approach could be expanded to urban infrastructures.

Metadesign is a non-teleological idea of design based on its own user's know-how. Computational media is explored to create a modifiable and malleable design by defining new connections between users and systems. Metadesign interactions concern releasing processes that surpass the instrumental use of technology. Participation and flexibility work to transform the design environment by establishing spontaneous and autonomous relationships between users and systems. These connections are based on a shared construction of the environment within which interaction triggers the emergence of new mentalities (Giaccardi, 2003). The design from this perspective intentionally gives space for users to contribute with their visions and goals, not only at design time but throughout the use. To embrace participation at use time requires more than knowing the context or attending to users' emergent needs. It is necessary to design immersive systems in such a way that users can express themselves and engage in activities that are truly meaningful to them (Fischer \& Giaccardi, 2004).

The systems created, therefore, should be classified as socio-technical systems that are formed by two fundamentally different subsystems: the social and technological ones. They are based on the idea that technology alone is not capable of improving people's social structures and behavior (Gerhard Fischer \& Herrmann, 2011). Their design must consider the interactions and dependencies that are related to continuous evolution, and that is influenced by conflicts of interest and power relations between these two subsystems (Herrmann, 2009). From this approach, systems are designed to guarantee more than integration 
between them, but also to support each other, resulting in a better performance of both (Gerhard Fischer \& Herrmann, 2011). We could affirm that in relation tosociotechnical systems, the two subsystems' integration will occur at the moment of use, but from Metadesign perspective, these systems must focus on what can emerge from them and contribute for mutual evolution. The design must result in open systems, in which are given opportunities and tools to users create their customizations, letting systems meet their needs and extend a social reward to them.

Metadesign sees creativity as a phenomenon that can happen in daily problem-solving activities, and when explored in design it can reach the form of social creativity called co-creation (Giaccardi, 2003). Co-creation is related to creations that emerge in relational sociotechnical environments from a process of sharing solutions with value and meaning. It is related to the interpretation, synchronization, and improvisation triggered by the emotions of a situated experience (Gerhard Fischer et al., 2005). In Metadesign each action of interaction is an act of creation (Giaccardi, 2003), supporting both the individual and social aspects, and finally the evolution of the sociotechnical system itself.

Metadesign brings the following conditions to sociotechnical environments: 1. they must be flexible because the use conditions cannot be completely predicted; 2. they should be improved by user collaboration; 3 . they must be designed to evolve (Gerhard Fischer \& Giaccardi, 2004). The system must be designed as an unfinished system to achieve these goals, and to that, it must be seen as a mean, not an end. The primary focus must be its openness. This feature will embrace continuous changes, and the status of "continuous beta" will become a desirable attribute of what is called an open system(Gerhard Fischer, 2009).

While Metadesign embraces participation to respond to anticipation issues, participation can also be seen as a possible approach to deal with complex design problems and long-term projects. These complex situations require greater knowledge than a single person can possess(Gerhard Fischer et al., 2005) and longer development. Bottom-up approaches to urban infrastructures design could be framed inside these categories especially because of its multiple users'condition. We could see the bottom-up structures as social knowledge constructed collectively from a series of individual knowledge and extended over time. Inside the system, both types of knowledgeshould be integrated by creating models of collaboration that support sociotechnical systems natural evolution through the assimilation of individual and social creativity. In this case, it is necessary to look at long-term collaboration as crucial for a systems' development (Gerhard Fischer, 2010).

The necessity to incorporate tacit knowledge related to multiple user's know-how also emerges supported by the concept of co-creation. Individually this knowledge is related to intuition and the ability to do something without necessarily being able to explain it. When referring to a group, tacit knowledge is related to practices that arise from interaction, and that is built over time (Gerhard Fischer et al., 2005). The challenge of expressing tacit knowledge when related to a collective construction can focus on the interactions emerging within the system. Therefore, these systems require the creation of means to express and communicate users' creativity.

The communication inside Metadesign also requires knowing which group of actors are related to the design problem. In the case of urban infrastructures design, we can identify at least two: the designers and users. Looking at these groups from the idea of two different Communities of Practice (COPs) integrating a Community of Interest (COI) (Fischer \& Ostwald, 2003)we consider the need to ensure communication between designers and users when designing these systems. COPs are usually understood as communities of professionals in the same area who usually know the answer to a specific problem, while COls are formed by professionals from different areasworking towards the same end. This approach sees both as knowledge bearers in the task of solving the same problem. In our research, we will see designers and users as members of a $\mathrm{COI}$ and their knowledge as the basis for creating a bottom-up structure. To this purpose, we need to establish communication inside this COI. This task requires the creation of common points of interest among these interlocutors. Within systems, these points must be achieved concretely or abstractly, configuring themselves as matter or concepts, through boundary objects(Gerhard Fischer et al., 2005). These objects are based on shared knowledge among communities, allowing ideas to be externalized. It facilitates communication and understanding beyond a temporal, conceptual and technological dimension. However, it is important to highlight that thecommunication of knowledge, and also co-creation, arises around the interaction with these objects and not from the objects itself (Gerhard Fischer \& Bell, 2004). It is when these objects are used and evolve that they support the sociotechnical system evolution as a whole (Fischer \& Ostwald, 2003).

Finally, to allow multiple users' participation, focusing on unexpected events occurring within these complex systems, asynchronous communication must be incorporated (Gerhard Fischer et al., 2001) creating what we believe to be the one of the basis for new approaches to urban infrastructures.

\section{METHODOLOGY}

The project draws inspiration from the previously mentioned problems in urban lighting design and is based on the presented Metadesign conceptual framework. We hypothesize that a system based on this mode of design can bring breakthroughs that could cope with ill-defined problems in urban lighting design. To address these shortcomings, our methodological approach is experimental and we are developing a system based on Metadesign principles. We believe that from this proposal we will be able to suggest the design of systems open to collaboration through interaction at use time, supporting new forms of interaction and the emergence of creativity and evolution. This suggestion will allow us to advance the discussion around the design and development of new tools in lighting design andalso to explore the influence and the implications of Metadesign in a bottom-upurban infrastructure. Here we will describe the current research effort to develop the system design. With the description, 
we intend to inform which tools we are using to frame the problem.

When facing the problem, we decided to propose an urban lighting system for pedestrian areas embracing the existing gap of lighting guidelines for public open spaces inBrazilian standards (Quintanilha, 2015). To develop our experiment, we will base our decisions on the idea that desirable lighting is directly related to its context and to the main activities people perform within their environment (Offermans, 2016). From that, we will propose a system to approach theproblem of designing urban lighting systems for public open spaces thatare areas with insufficient guiding information. For this purpose, we will follow the process model in support of Meta-Design: SER Model Seeding, Evolutionary Growth, Reseeding Model (Fischer et al., 2001). This model is an emerging descriptive and prescriptive approach that supports system design for best fitting an emerging and evolving context (Eden, Fischer, Giaccardi, \& Ye, 2007). Our research is located inside the seeding process, in which a seed is created as an initial collection of knowledge that is designed to be open and evolve at use time (Fischer \& Giaccardi, 2004).

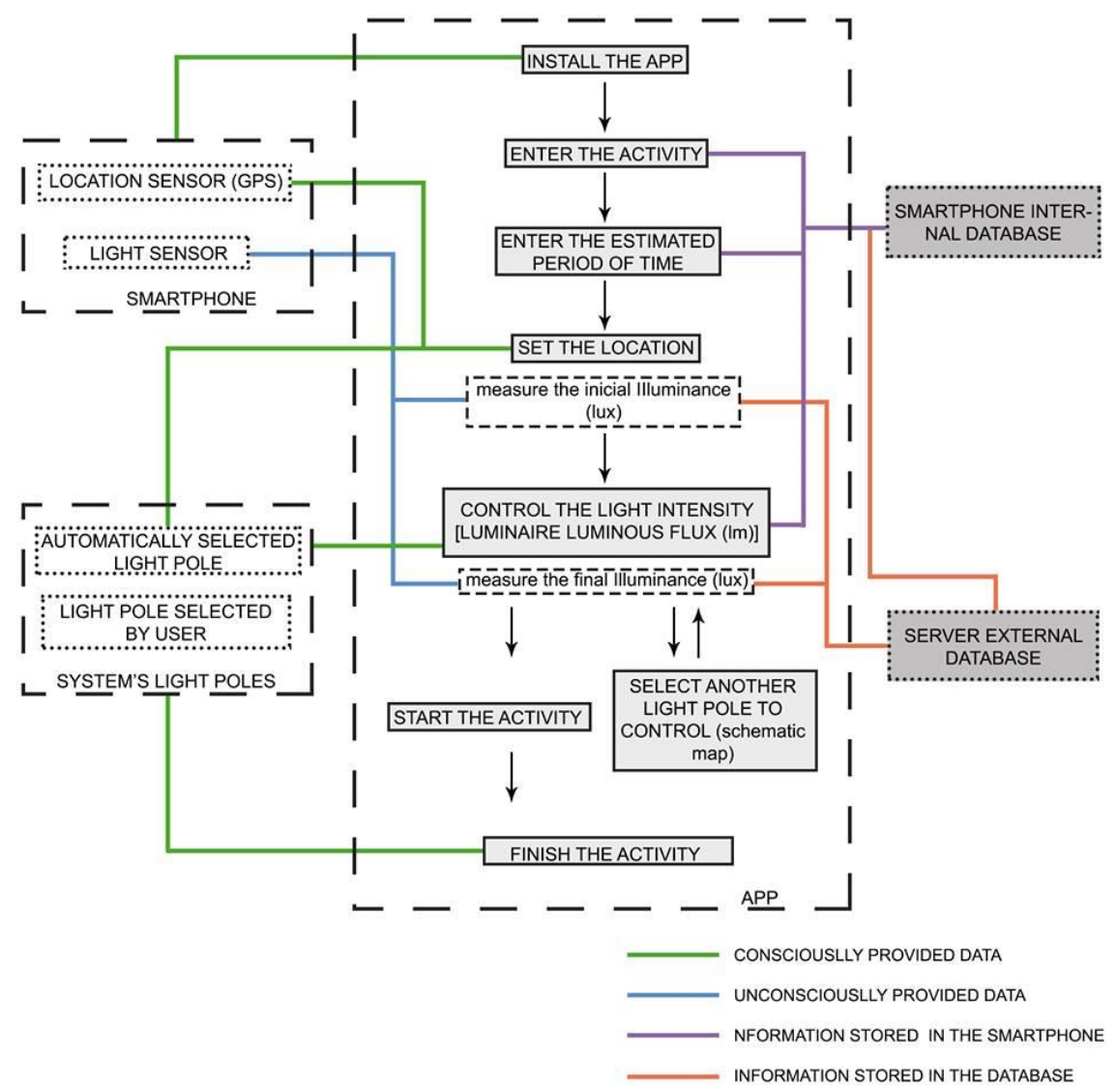

Figure1: System operation process chart. Source: Authors.

\section{DESCRIPTION}

A hypothetical terrain was createdwith dimensions close to $25 \mathrm{~m} \times 50 \mathrm{~m}$, where six lighting fixtures were located in two rows, simulating light poles. The user' interaction with the system would happen through a smartphone application designed with the visual programming environment MIT App Inventor. Using the app people will be able to control the light intensity of these luminaires according to their requirements to attend the activities they will be performing inside the space.

For operating the application, the smartphone must be equipped with both lighting and location sensor. After installing the app, when the user reaches the area and runs the app it is necessary to enter the information about the activity he or she will perform and the estimated period of time. This data will be stored in the smartphone's internal database and in the system's external database. The first could be used as a starting point for nextuses, and the second, with multiple users inserting this information over time, could be used to identify patterns of use within the system.

After entering this information, the application will run, and the user will be asked to position himself in their favorite location. At this point through the GPS location sensor, their geographic coordinates will be gathered by the system. This information is given by the user consciously. At the same time, when pressed this button, the lighting sensor will measure the Illuminance level at that location, typifying unconscious information gathered. The GPS location will be sent to the lighting control system that will determine which lighting fixture is closest to the user. This lighting pole will be activated and will be ready to be 
controlled. The unconsciously provided data of Illuminance value will be saved in the external database.

The automatically selected lighting fixture will be ready to be controlled by the user through a slider. This slider works like a dimmer switch, on the touchscreen of the smartphone. After changing the light intensity, if the user is satisfied, the new Illuminance value will be collected by the smartphone and saved in the external database. If the user finds necessary to manipulate any other luminaire around him, a schematic map will open, and he will be able to select through the touchscreen a new light pole to control the light.

When the configuration is completed, the user can start practicing their activity. Personal settings will be reset at the end of this user' activity, either automatically, when the estimated time interval ends, or voluntarily, by a button.

\section{SCREEN 1: INSERTING ACTIVITY RELATED DATA}

The user's first contact with the application interface is through Screen 1, from which the user will start entering the requested data in each field, over five steps. In each step, users will provide information related to: 1 st. The activity that he will perform; 2 nd. The amount of time he will take to perform such activity; 3rd. His location inside the space in geographical coordinates (latitude and longitude) and light intensity; 4th. The value of the desired illuminance index, according to their need; 5 th. The end of the activity, by pressing the button "OK", when finished.

Screen 1 (Figure2) is composed of 4 visible components (label, textbox, spinner, and button - as named by MIT App Inventor) and an invisible component, called tinyDB. The label contains an explanatory text, whose function is to guide the user about the subsequent step. First, this component instructs the user to freely write on the textbox the activity that he or she will perform, such as reading, doing yoga, or playing soccer, among others. After this step, there is a second label that guides the user to select from a list of predefined elements, called spinner, the time interval he will be performing the activity, for example: $15 /$ $30 \mathrm{~min}, 30 / 45 \mathrm{~min}, 45 / 60 \mathrm{~min}$, and so on. The "SAVE PRESETS" button is present on all the screens of the application and is linked to the tiny $D B$ component, whose function is to store information in a local database. In this way, when initializing the application after it has been closed, or when returning to a particular screen, the data will be displayed in their respective fields with the information previously provided by the user. Then, through the "NEXT" button, the user moves to the next screen of the application (Screen 2).
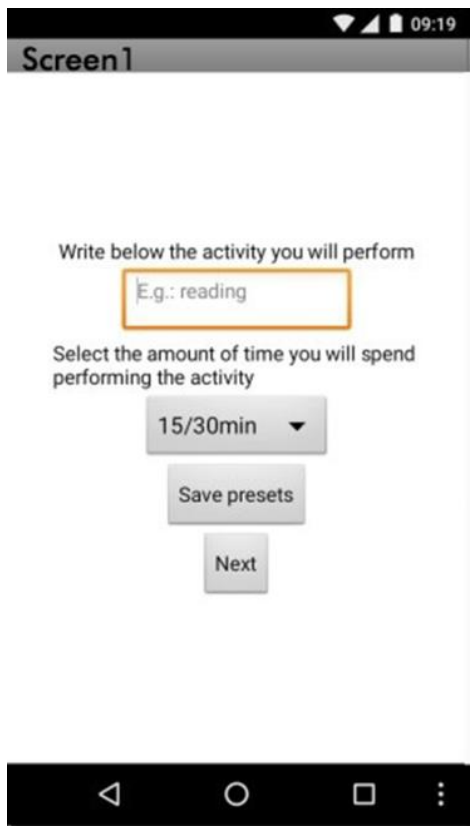

Figure 2: Screen 1: Insert the activity. Source: Authors.

\section{SCREEN 2: COLLECTING SPACE LOCATION DATA}

A pop-up notification appears on the screen, telling users that their smartphone's GPS must be enabled for the application to work. The user will be instructed to position himself where the action will be performed (Figure 3). His coordinates will appear on the screen, and by clicking the "SAVE LOCATION" button, his location will be sent to the database. This data will besent by users to the database, along with the date, time and initial Illuminance level detected by the smartphone. When this data is saved, a pop-up confirmation appears (Figure 4), redirecting users to Screen 3.

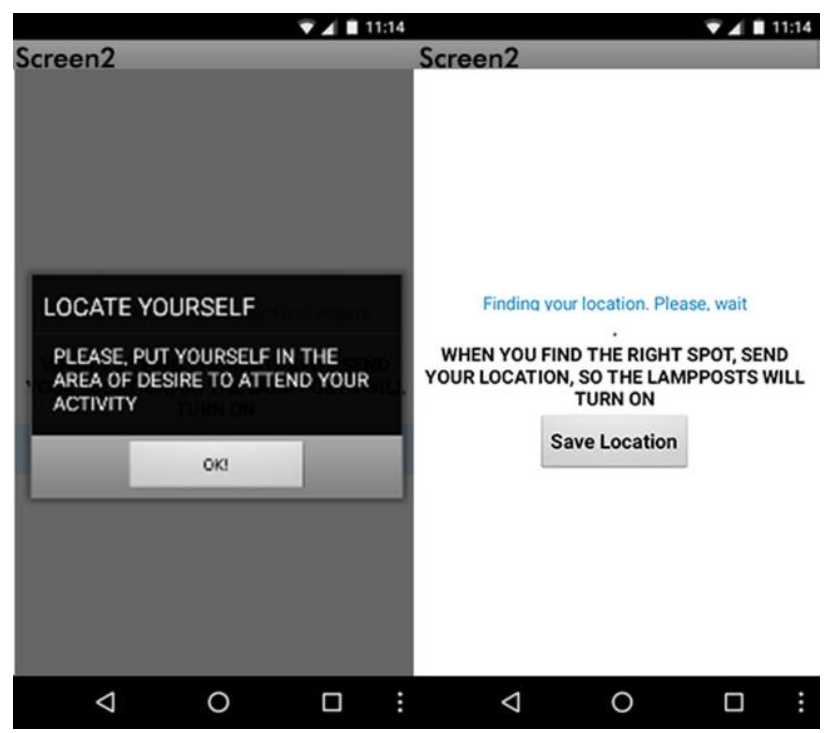

Figure3: Screen 2: Finding user location. Source: Authors.

The coordinates will be used to select automatically the closest lighting fixture to be controlled. We believe that the data related to the illuminance collected without user knowledge will help to identify patterns related to specific activities and their corresponding Illuminance value. 


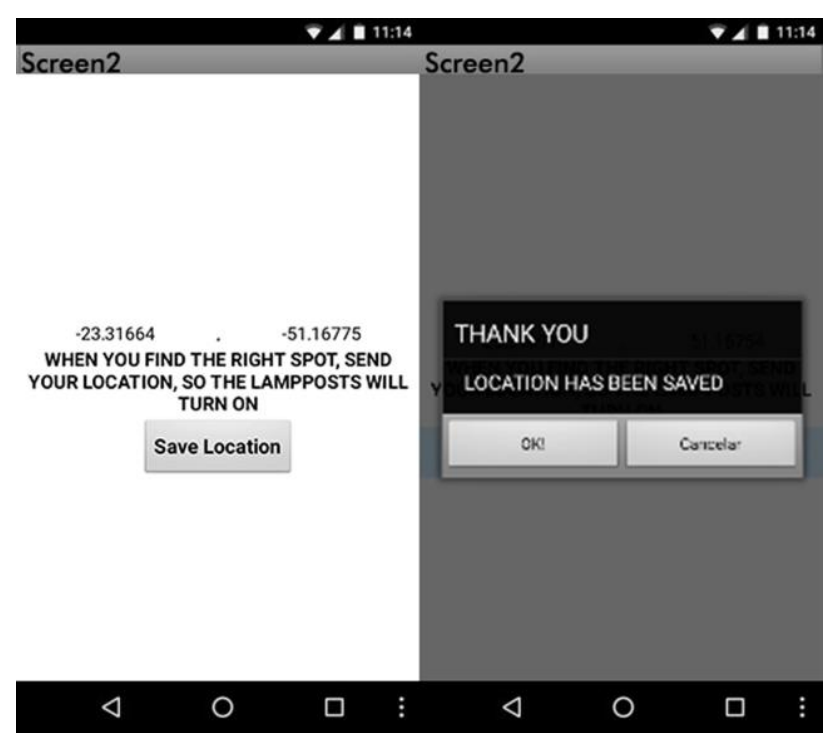

Figure4: Screen 2: Saving coordinates. Source: Authors.

\section{SCREEN 3: ADJUSTING LIGHTING INTENSITY}

Screen 3 consists of a label that instructs users to adjust the luminous flux index of a given luminaire by manipulating the slider. This function is a progress bar that contains a draggable button, whose positions are equivalent to luminous flux values, limited to a minimum and maximum pre-set indexes. In this way, the user can touch the button and drag it left or right to set the position of the slider button, and consequently control the light intensity. The "BACK" button, located at the top of the screen, when triggered, returns the user to the previous application screen. The "OK" button, located at the bottom, when pressed, triggers two actions: the first one is to change to the next screen, and the other one is to capture the Illuminance value (using the lighting sensor) after its adjustment by the user.

This function will be related to the perceived light intensity each user believes to be the best to perform specificactions. This data will complement the information about patterns of use related to specific activities and Illuminance. We believe that this tool will provide the opportunity for users to better perform their activities supported bybetter lighting levels and that the openness to their participation will create a feeling of connection with their environment.

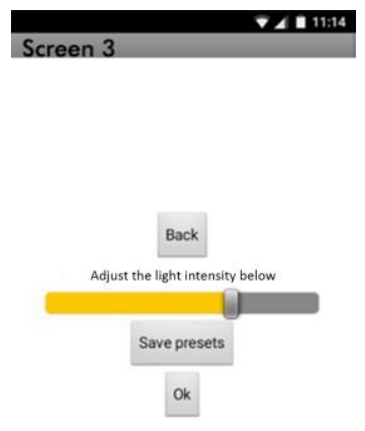

Figure5: Screen 3: Controlling light intensity. Source: Authors.

\section{SCREEN 4: CONTROLLING MORE LIGHTING FIXTURES}

If the user is dissatisfied with the maximum intensity of light to be reached on Screen 3, he will be redirected to a screen with a schematic map representing the public open space and the light poles inside it. In this map, it will be possible to see his location, the active lamppost,and the others within the space (Figure 6). The user can then select a new lamppost to be controlledby touching it on the screen, and then he is redirected to Screen 3.

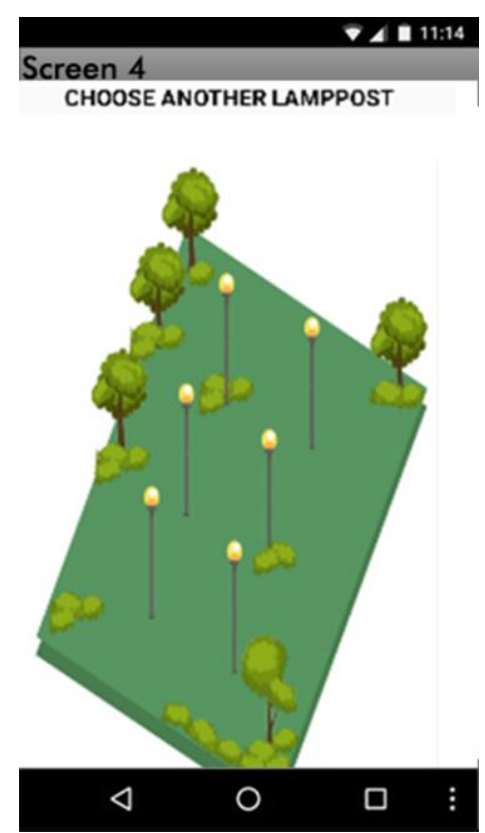

Figure6: Screen 4: Choosing another lamppost. Source: Authors.

\section{SCREEN 5: FINISHING THE ACTIVITY}

A pop-up notification will appear on the screen, warning that the time interval set on Screen 1 is finishing. A warning will sound, indicating that the lighting set defined on Screen 3 will reset to the initial system configuration (Figure 7). The option to finish the activity before the predetermined time is possible through the "FINISH ACTIVITY" button (Figure 6) and the user will receive a reset confirmation of the luminaire being controlled (Figure8).

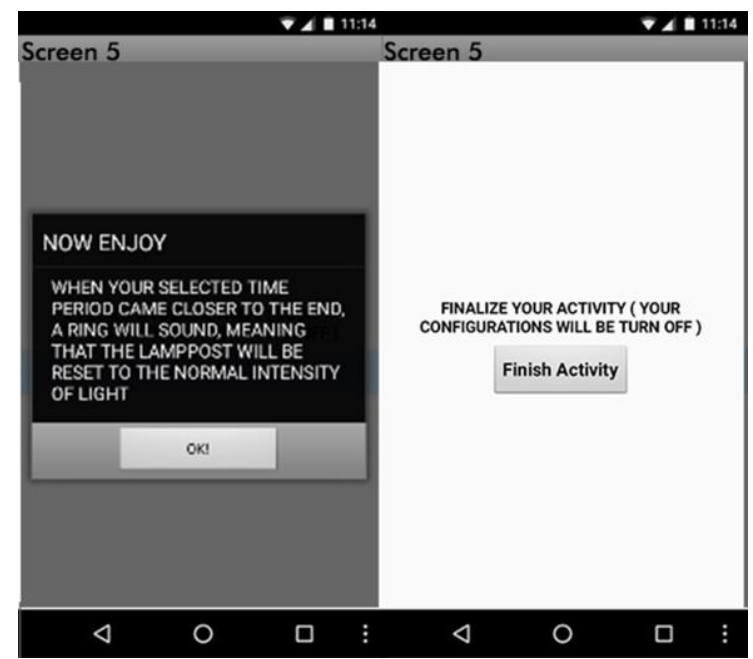

Figure7: Screen 5: Finishing the activity. Source: Authors. 


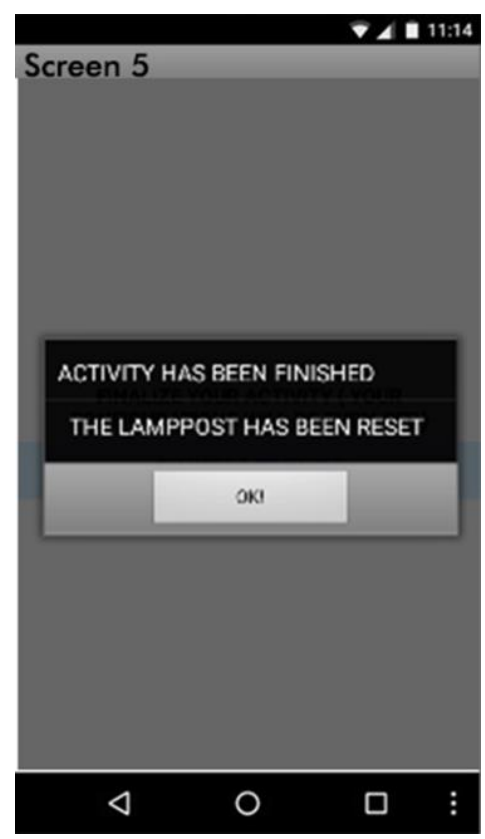

Figure8: Pop up Screen 5. Source: Authors.

\section{ASSESSMENT}

So far, our initial assessments of the ongoing research are revealing that Metadesign framework can be useful to address problems in urban lighting design. The design of a system open to participation at use time appears to be an alternative to respond to:

- The problem of designing lighting systems for public open spaces, that are areas with insufficient guidelines, since the capacity of collecting data related to patterns of use cannot be entirely predicted at design time, especiallyrelated with numerous possibilities of use;

- The problem of designing lighting systems for multiple users because of the wide variety of activities and preferences related to different people;

- The potential of personalizing the luminaire's luminous flux and support better performance in relation to better lighting levels, incorporating issues closer to their context of use;

- The potential of personalizing the luminaire's luminous flux and create a feeling of connection with the environment. Users will engage in personally meaningful actions, and a relational sociotechnical environment will be created. Within these environments, solutions will be shared through different light settings. We believe that these environments will be able to engage people in making choices triggered by their emotions in a situated experience.

- The idea of building cities through a more democratic perspective with users collaborating with data related to their emergent needs, and also because the openness of these systems gives opportunities to personalized settings.

\section{CONCLUSION}

Our research project illustrates an unique approach towards urban lighting design, creating foundations for designing lighting systems open to participation through interaction at use time. Our approach provides an alternative framework to the existing problems that infrastructure design is facing in the current context. For now, Metadesign seems to be an option to respond to the main problemsof: 1. anticipation - the need to incorporate issues closer to context and use; 2. participation - the construction of cities through a bottom-up perspective; 3 . socio-technical - the inclusion of a user perspective into these systems.

To propose solutions from this non-traditional design perspective require a balance between system's openness and the limitation of its fixtures. Because ICT is now embedded on infrastructures, it is possible to think about urban lighting design through a Metadesign perspective. However, this field of application still haslimited possibilities of changing their material portion. The malleability considering the majority of LED luminaires are restricted to changes in intensity, color or orientation. This leads to an effort concentrated mostly on designing the interactions that are not materially related to the environment, but to the interaction among users themselves and with the system. These last forms of interactions will define the way the environment will be perceived and experienced.

Another feature that has imposed difficulties is the openness of the system. Firstly when related to the idea of accommodating multiple users'emergent needs, it requiresthedefinition of some constraints. These restrictions at this point, seems to move towards the definition of hierarchies among users. Secondly, the openness when related to evolution represents a higher degree of openness than designing only for emergent needs. At last, the openness related to user participation in the moment of use can bring to the design a feeling of uncertainty related toMetadesignintentionally abandon of some control. Participation at use time requires a different attitude from designers configuring in some degree a real non-teleological mode of design.

Despite these difficulties, this research is working to contribute to a better understanding of these intricated relations among social, technical, material and methodological aspects that are equally essential to the accomplishment of Metadesign based systems. All these statements are preliminary reflections from our work and do not constitute the final proposal of this research. The experiment development so far is mostly concentratedon capturing individual knowledge and triggering individual creativity. The identification of real boundaries and pitfalls associated with the system functionality and with Metadesign framework will depend on further development.

\section{ACKNOWLEDGMENTS}

We thank to Guilherne Massahiro Rodrigues Yuki and Professor Ernesto Ferreyra Ramírez for technical support in Arduino. We thank too National Council for the Improvement of Higher Education (CAPES) for the scholarship in support of this research. 


\section{REFERENCES}

Eden, H., Fischer, G., Giaccardi, E., \& Ye, Y. (2007). Report on NSF science of design team project: "A meta-design framework for participative software systems." 2nd International Conference on Design Science Research in Information Systems and Technology, DESRIST 2007, 272 291.

Fischer, G. (2009). End-User Development and Meta-design: Foudations for Cultures of Participation. 2nd International Symposium, IS-EUD 2009.

Fischer, G. (2010). Extending boundaries with meta-design and cultures of participation. Proceedings of the 6th Nordic Conference on Human Interaction: Extending Boundaries, 168-177. https://doi.org/10.1145/1868914.1868937

Fischer, G., \& Bell, A. G. (2004). Social Creativity: Turning Barriers into Opportunities for Collaborative Design. Proceedings of the Eighth Conference on Participatory Design: Artful Integration: Interweaving Media, Materials and Practices, 1, 152-161.

Fischer, G., \& Giaccardi, E. (2004). Meta-design: A Framework for the Future of End-User Development. End User Development. Human-Computer Interaction Series, 9, 427457. https://doi.org/10.1007/1-4020-5386-X_19

Fischer, G., Giaccardi, E., Eden, H., Sugimoto, M., \& Ye, Y. (2005). Beyond binary choices: Integrating individual and social creativity. International Journal of Human Computer Studies, 63(4-5 SPEC. ISS.), 482-512. https://doi.org/10.1016/j.jhcs.2005.04.014

Fischer, G., Grudin, J., McCall, R., Ostwald, J., Redmiles, D., Reeves, B., \& Shipman, F. (2001). Seeding, Evolutionary Growth and Reseeding: The Incremental Development of Collaborative Design Environments. Coordination Theory and Collaboration Technology, 447-472. https://doi.org/doi: 10.1145/191666.191770

Fischer, G., \& Herrmann, T. (2011). Socio-technical Systems: A Meta-design Perspective. International Journal of Sociotechnology and Knowledge Development, 1-33.

Fischer, G., \& Ostwald, J. (2003). Knowledge Communication in Design Communities. Barriers and Biases in ComputerMediated Knowledge Communication, 1-32. https://doi.org/10.1007/0-387-24319-4_10
Gaston, K. J., Gaston, S., Bennie, J., \& Hopkins, J. (2014). Benefits and costs of artificial nighttime lighting of the environment. Environmental Reviews, 23(1), 14-23. https://doi.org/10.1139/er-2014-0041

Giaccardi, E. (2003). Principles of Metadesign Processes and Levels of Co-creation in the New Design Space.

Giaccardi, E. (2005). Metadesign as an emergent design culture. Leonardo, 38(4), 342-349. https://doi.org/Doi $10.1162 / 0024094054762098$

Giaccardi, E., \& Fischer, G. (2008). Creativity and evolution: A metadesign perspective. Digital Creativity, 19(1), 19-32. https://doi.org/10.1080/14626260701847456

Herrmann, T. (2009). Systems Design with the Socio-Technical Walkthrough. Handbook of Research on Socio-Technical Design and Social Networking Systems, 336-351. https://doi.org/10.4018/9781605662640.ch023

Magielse, R., Hengeveld, B. J., \& Frens, J. W. (2013). Designing a light controller for a multi-user lighting environment. lasdr 2013, 2811-2822.

Nabian, N., \& Ratti, C. (2012). Top-Down/Bottom-Up Urbanism. Inscribing a Square: Urban Data as Public Space, 215-236. Retrieved from http://senseable.mit.edu/papers/pdf/20120801_Nabian_Ratti_ TopdownBottomup_InscribingSquare.pdf

Nabian, N., \& Ratti, C. (2013). A Collaborative approach to architecture. https://doi.org/http://dx.doi.org/10.1371/journal.pone.0149607

Offermans, S. A. M. (2016). Interacting with Light.

Quintanilha, I. de C. (2015). A influência da iluminação artificial no comportamento de usuários de praças públicas: O caso da Praça Coronel Pedro Osório.

Roche, S., Nabian, N., Kloeckl, K., \& Ratti, C. (2012). Are "Smart Cities "Smart Enough? GSDI World Conference, 215-236.

Shaw, R. (2014). Street-Lighting in England and Wales: New Technologies and Uncertainty in the Assemblage of StreetLighting Infrastructure. Environment and Planning, A, 46(9), 2228-2242. https://doi.org/10.1063/1.2756072 